\title{
Anti-transcription intermediary factor 1 gamma (TIF1 $\gamma$ ) antibody-positive dermatomyositis associated with ascending colon cancer: a case report and review of the literature
}

Ryohei Ono ${ }^{{ }^{*}} \mathbb{D}$, Tomohiro Kumagae ${ }^{1}$, Mari Igasaki ${ }^{1}$, Takaaki Murata ${ }^{2}$, Masaki Yoshizawa $^{3}$ and Izumi Kitagawa ${ }^{1}$

\begin{abstract}
Background: Anti-transcriptional intermediary factor 1 gamma (TIF1 $\gamma$ ) antibody is a marker for predicting cancer association in patients with dermatomyositis (DM). The overall survival rate in DM patients with cancer was reported to be considerably worse than that in DM patients without cancer. However, the treatment for cancer-associated DM remains controversial, because the treatment priority between surgical resection for the tumor and internal treatments, including glucocorticoids, immunosuppressive agents, and intravenous immune globulin, has not been established.
\end{abstract}

Case presentation: We report the case of a 57-year-old Japanese man diagnosed with anti-TIF1Y antibody-positive DM associated with ascending colon cancer. His clinical symptoms included facial and brachial edema, muscle weakness, dysphagia, myalgia, and rash. Physical examination revealed periorbital edema and Gottron's papules over his knuckles with brachial edema, and tenderness and weakness of the proximal limb muscles. The findings of hyperintense muscles in T2-weighted sequences of brachial contrast-enhanced magnetic resonance imaging and the infiltration of lymphocytic cells and CD4-positive lymphocytes from muscle biopsy were compatible with the diagnostic criteria for dermatomyositis. Anti-TIF1Y antibody was positive by immunoprecipitation assay. He first started internal treatment including intravenous immunoglobulin, steroid pulse, prednisolone, and azathioprine, followed by surgical resection for the tumor because of the elevation of creatine kinase and progression of dysphagia. However, clinical symptoms did not improve, and the patient died 6 months later.

Conclusions: We faced difficulties in determining the treatment priority between surgical resection and internal treatment for our case; therefore, this case would be educational for readers. We searched PubMed to identify Englishlanguage case reports of anti-TIF1Y antibody-positive dermatomyositis with malignancy and found 21 reported cases. We herein review and summarize previously reported cases of anti-TIF1Y antibody-positive DM with malignancy. Cancer screening is essential in patients with anti-TIF1Y antibody-positive dermatomyositis because it is associated

*Correspondence: ryohei_ono_0820@yahoo.co.jp

${ }^{1}$ Department of General Internal Medicine, Shonan Kamakura General

Hospital, 1370-1 Okamoto, Kamakura, Kanagawa 247-8533, Japan

Full list of author information is available at the end of the article original author(s) and the source, provide a link to the Creative Commons licence, and indicate if changes were made. The images or other third party material in this article are included in the article's Creative Commons licence, unless indicated otherwise in a credit line to the material. If material is not included in the article's Creative Commons licence and your intended use is not permitted by statutory regulation or exceeds the permitted use, you will need to obtain permission directly from the copyright holder. To view a copy of this licence, visit http://creativecommons.org/licenses/by/4.0/. The Creative Commons Public Domain Dedication waiver (http://creativeco mmons.org/publicdomain/zero/1.0/) applies to the data made available in this article, unless otherwise stated in a credit line to the data. 
with a high prevalence of malignancies. Our review revealed that initial surgical treatment should be recommended for better prognosis if the general condition allows.

Keywords: Dermatomyositis, Anti-transcription intermediary factor 1 gamma, Anti-TIF1Y antibody, Cancer, Malignancy

\section{Background}

Dermatomyositis (DM) is an inflammatory myopathy characterized by skin rash and progressive, symmetrical weakness of the proximal muscles $[1,2]$. DM has been shown to be associated with malignant disease [3]. The overall survival rate in DM patients with cancer was found to be considerably worse than that in DM patients without cancer [4]. Recently, an anti-transcriptional intermediary factor 1 gamma (TIF1 $\gamma$ ) antibody was reported as a marker for predicting cancer association in patients with DM, since TIF1 $\gamma$, which regulates the tumor growth factor pathway, has been reported to be associated with tumor growth in some malignancies [5]. In a meta-analysis, Trallero-Araguas et al. reported that the pooled sensitivity of anti-TIF1 $\gamma$ antibody for diagnosing cancer-associated DM was $78 \%$, whereas specificity was $89 \%$ [6]. The treatment for cancer-associated DM remains controversial, because the treatment priority between surgical resection for the tumor and internal treatments, including glucocorticoids, immunosuppressive agents, and intravenous immune globulin, has not been established. We searched PubMed to identify English-language case reports of anti-TIF1Y

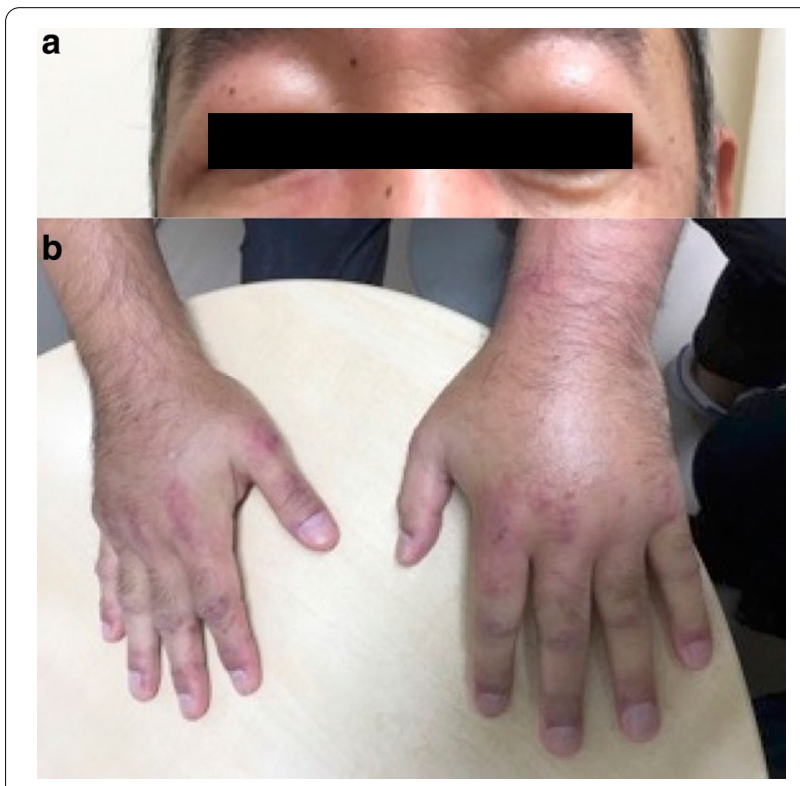

Fig. 1 Physical examination revealed periorbital edema (a) and Gottron's papules over his knuckles (b) antibody-positive dermatomyositis with malignancy and found 21 reported cases [7-27]. Herein, we report a case of anti-TIF1 $\gamma$ antibody-positive dermatomyositis associated with ascending colon cancer; previously reported cases of anti-TIF1 $\gamma$ antibody-positive dermatomyositis with malignancy are reviewed and summarized. This case may provide a unique perspective for readers and illustrate the difficulties in determining treatment priority between surgical resection and internal treatment.

\section{Case presentation}

A 57-year-old Japanese man presented with a 1-month history of progressive symptoms of facial and brachial edema, muscle weakness, dysphagia, myalgia, and a symmetrical widespread rash on his limbs and hands. He denied recent common cold symptoms. He was also noted to have unintentional weight loss $(3 \mathrm{~kg}$ over 1 month). His medical and family histories were unremarkable. He was diagnosed with type 2 diabetes mellitus 8 years ago, but he did not go to the hospital until this visit.

Vital signs showed that the patient was afebrile, with a heart rate of 90 beats per minute, blood pressure of $120 / 78 \mathrm{mmHg}$, normal respiratory rate, and oxygen saturation of $99 \%$ on room air. Physical examination revealed periorbital edema (Fig. 1a) and Gottron's papules over his knuckles (Fig. 1b) with brachial edema, and tenderness and weakness of the proximal limb muscles. Laboratory evaluation revealed elevated levels of creatine kinase (5002 U/L; reference range 30-175 U/L), aspartate transaminase (120 U/L; reference range, $12-35 \mathrm{U} / \mathrm{L})$, alanine aminotransferase (46 U/L; reference range 6-40 U/L), lactate dehydrogenase (440 U/L; reference range $119-229 \mathrm{U} / \mathrm{L})$, D-dimer $(9.1 \mu \mathrm{g} / \mathrm{mL}$; reference range $<1.0 \mu \mathrm{g} / \mathrm{mL}$ ), and hemoglobin A1c (9.2\%; reference range 4.6-6.2\%); however, white blood count, C-reactive protein, hemoglobin, electrolytes, lipid profile, and renal function were normal. Hepatitis B and C, and HIV serologies were all negative. Chest radiography showed no consolidation. Respiratory function tests, electrocardiogram, and echocardiogram were unremarkable. Because of the history and significantly elevated muscle injury biomarkers, we suspected inflammatory myositis. The patient underwent further evaluation to investigate the probable diagnosis.

Additional laboratory data demonstrated that antinuclear antibody was positive at 1:40 with a speckled 
pattern. In addition, anti-TIF1 $\gamma$ antibody was positive by immunoprecipitation assay, although other markers including anti-aminoacyl-tRNA synthetase, antimelanoma differentiation-associated gene 5 antibody, and anti-Mi2 antibody were negative. Brachial contrastenhanced magnetic resonance imaging (MRI) demonstrated hyperintense muscles in T2-weighted sequences

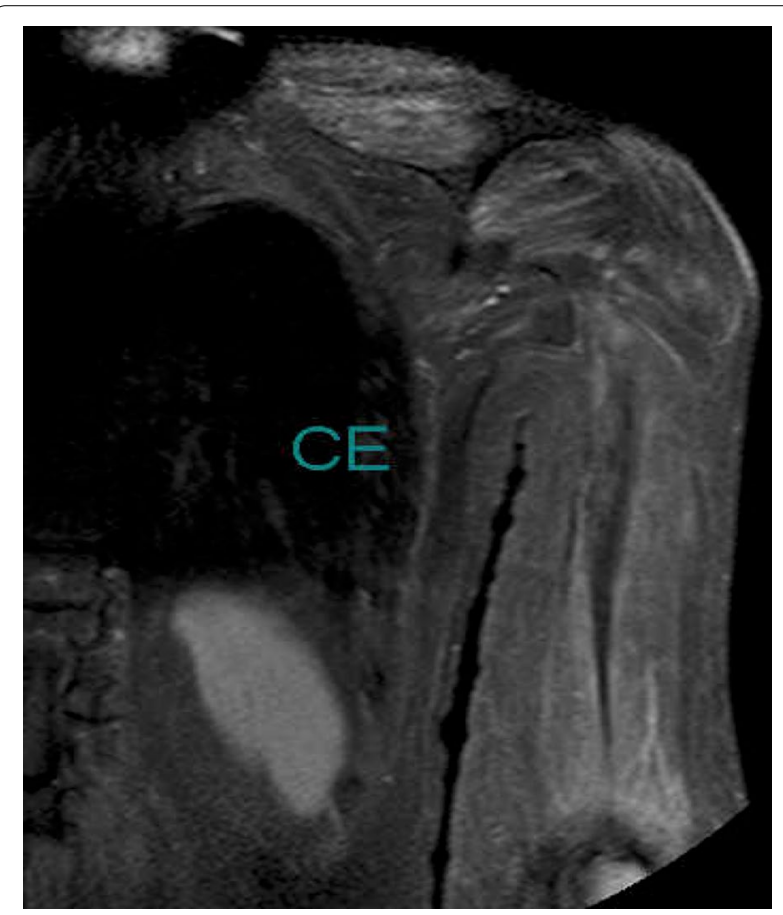

Fig. 2 Brachial contrast-enhanced magnetic resonance imaging demonstrated hyperintense muscles in T2-weighted sequences
(Fig. 2). A biopsy from the biceps brachii muscle was performed, and the infiltration of lymphocytic cells and CD4-positive lymphocytes was confirmed (Fig. 3). These findings were compatible with dermatomyositis. Since anti-TIF1 $\gamma$ antibody has been associated with malignancies in dermatomyositis patients, we performed a whole contrast computed tomography scan and endoscopy. Contrast computed tomography showed a tumor mass in the ascending colon with no other notable metastases (Fig. 4a). Colonoscopy revealed an ascending colon tumor (Fig. 4b). The histopathological findings of the biopsy from the ascending colon showed well-differentiated tubular adenocarcinoma (Fig. 5). A diagnosis of antiTIF1 $\gamma$ antibody-positive dermatomyositis with ascending colon cancer (cT4aN2M0, clinical stage IIIb) was made.

The clinical course is shown in Fig. 6. He was initially scheduled to undergo surgical resection for the ascending colon cancer after the definitive diagnosis; however, elevation of creatine kinase (15,667 U/L) and progression of dysphagia were noted before the operation. Thus, we decided that medical treatment should be performed first. Subsequently, intravenous immunoglobulin (IVIG) and $1 \mathrm{mg} / \mathrm{kg}$ of prednisolone with slow tapering of the dose was started from day 40, and the level of creatine kinase decreased significantly. However, his dysphagia did not improve, and creatine kinase was elevated again on day 57 . Thus, $1 \mathrm{~g} /$ day of methylprednisolone (mPSL) for three consecutive days was administered from day 58 . After the second cycle of IVIG (on day 75) and steroid pulse therapy (on day 81), $50 \mathrm{mg} /$ day of azathioprine was started on day 97 because creatine kinase was decreased but the dysphagia persisted. As the patient's condition had deteriorated, manual muscle testing of his limb was

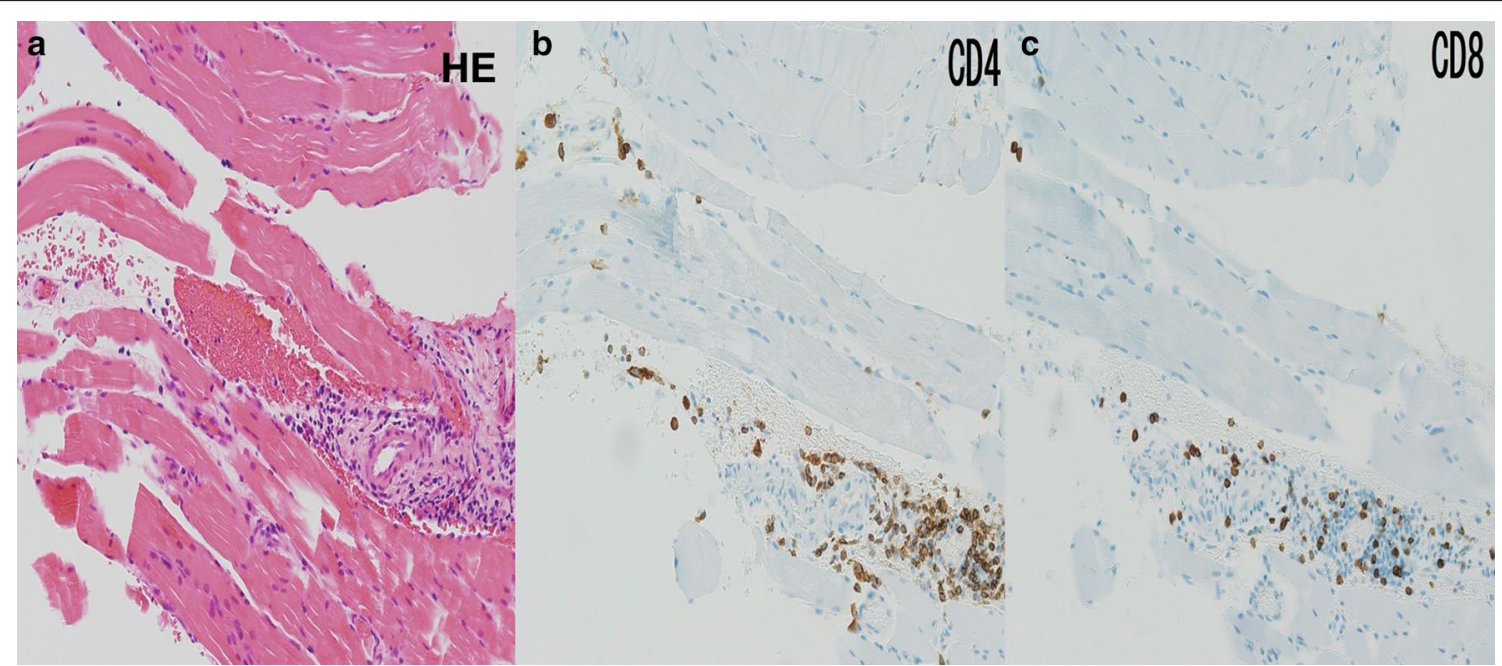

Fig. 3 a Hematoxylin and eosin stain ( $\times 40$ magnification) of the muscle showing the infiltration of lymphocytic cells. b CD4 antibody staining ( $\times 100$ magnification) and c CD8 antibody staining (×100 magnification) confirmed the predominant presence of CD4-positive lymphocytes 


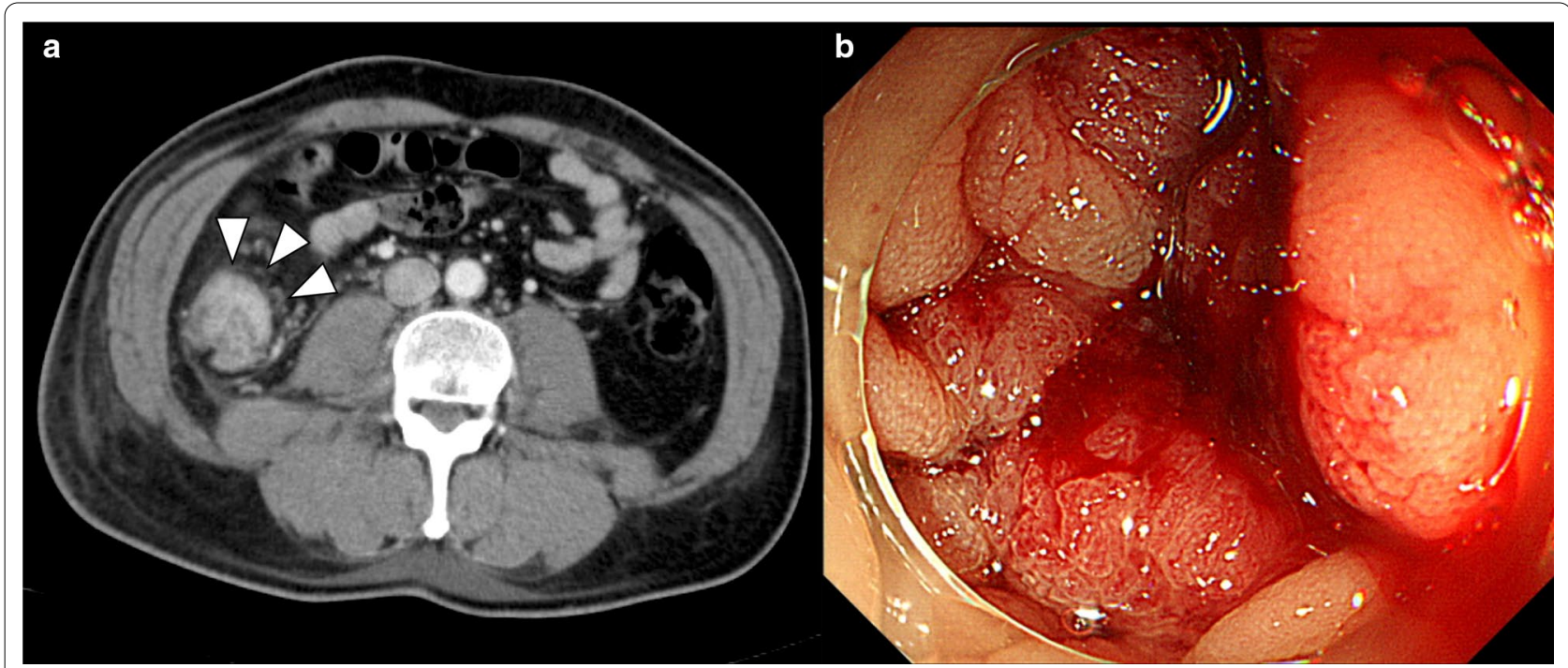

Fig. 4 Colonoscopy revealing the ascending colon tumor. Contrast computed tomography showing a tumor mass in the ascending colon (white arrows) with no other notable metastases (a), and colonoscopy revealing an ascending colon tumor (b)

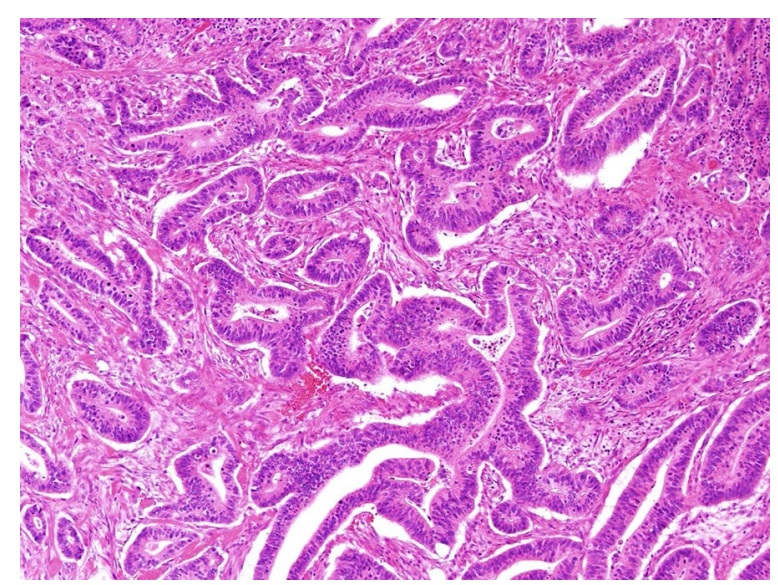

Fig. 5 The histopathological findings of the biopsy from the ascending colon showing well-differentiated tubular adenocarcinoma (hematoxylin and eosin stain, $\times 400$ magnification)

grade 2 out of 5 , and medical treatment was considered ineffective, PSL was increased from 15 to $20 \mathrm{mg}$ per day and surgery including right hemicolectomy, gastrostomy, and tracheostomy was performed on day 124 .

After surgery, the patient was almost bedridden due to disuse syndrome despite continuous rehabilitation. Fever was noted on day 146, and broad-spectrum antibiotic therapy was not effective. Further investigation revealed positive serum cytomegalovirus antigen levels. Administration of ganciclovir $300 \mathrm{mg} /$ day was initiated, but further complication of melena was noted. Gastroscopy was performed and showed cytomegalovirus esophagitis (Fig. 7). The patient died 6 months later (204 days) after hospitalization due to the progression of uncontrollable infection.

\section{Discussion}

The prevalence of malignancy in patients with dermatomyositis is estimated at approximately $20-30 \%$ [28]. An anti-TIF1 $\gamma$ antibody associated with malignancy has been identified in dermatomyositis [7]. This antibody is confirmed in approximately $20 \%$ of adult patients with dermatomyositis, and $60 \%$ to $90 \%$ of these patients have malignant disease $[6,28]$. The treatment order for cancer-associated DM has not been established, especially whether internal treatment or surgical resection should occur first. To our knowledge, this is the first reported case of anti-TIF1 $\gamma$ antibody-positive dermatomyositis associated with colon cancer.

The major clinical features of the 21 previously reported cases of anti-TIF1 $\gamma$ antibody-positive dermatomyositis associated with cancer and our case are summarized in Table 1 [7-27]. We also investigated the relationship between outcome and treatment, since we faced difficulties in determining the treatment priority between surgical resection and internal treatment. The mean age ( \pm standard deviation) of the population was $63.7 \pm 13.7$ years (range, 22-83 years), of whom 12 were male and 10 were female. The most common presenting symptom was rash $(86 \%)$, followed by muscle weakness (50\%), dysphagia (45\%), facial edema (14\%), and myalgia (14\%). Although all cases were associated with concurrent DM and cancer, initial diagnosis of DM (68\%) was more prevalent than that of cancer (32\%). Most of the cases were single cancer; however, two cases of double 


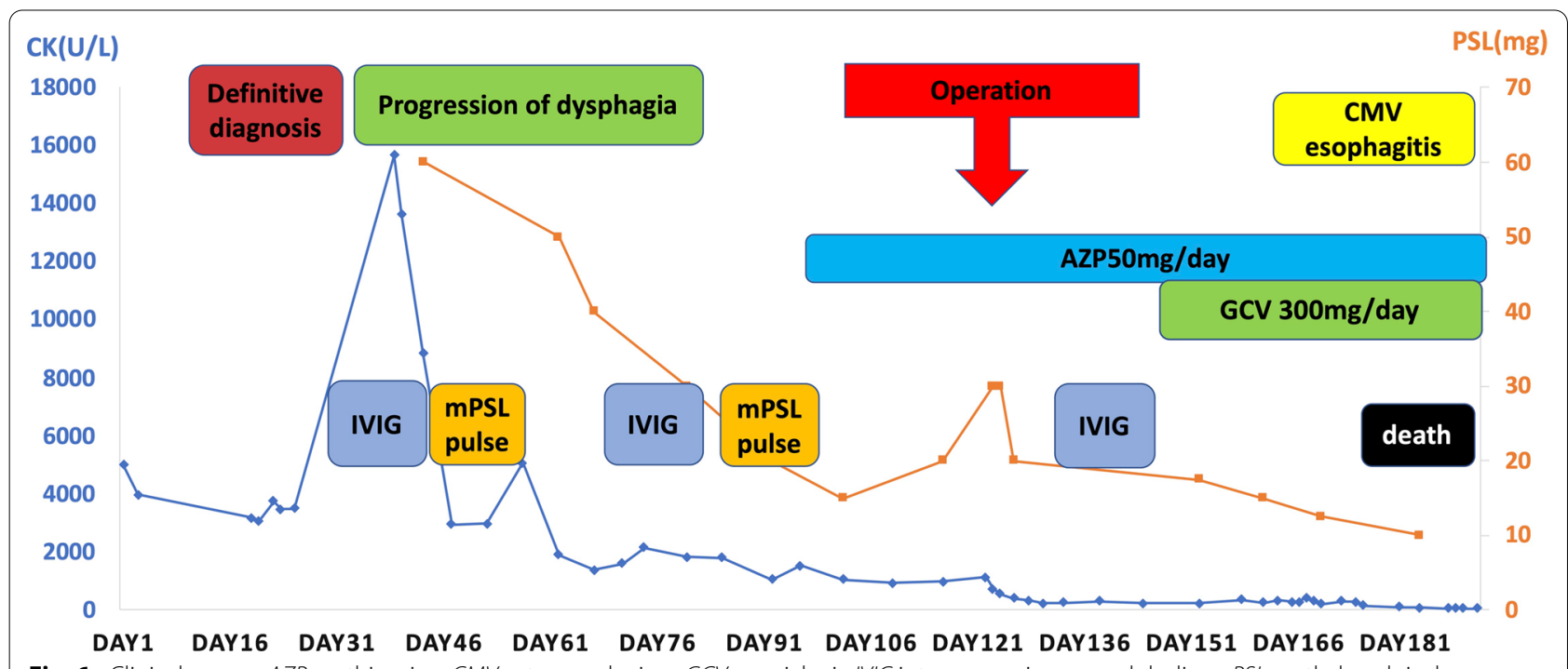

Fig. 6. Clinical course. AZP azathioprine, CMV cytomegalovirus, GCV ganciclovir, IVIG intravenous immunoglobulin, mPSL methylprednisolone.

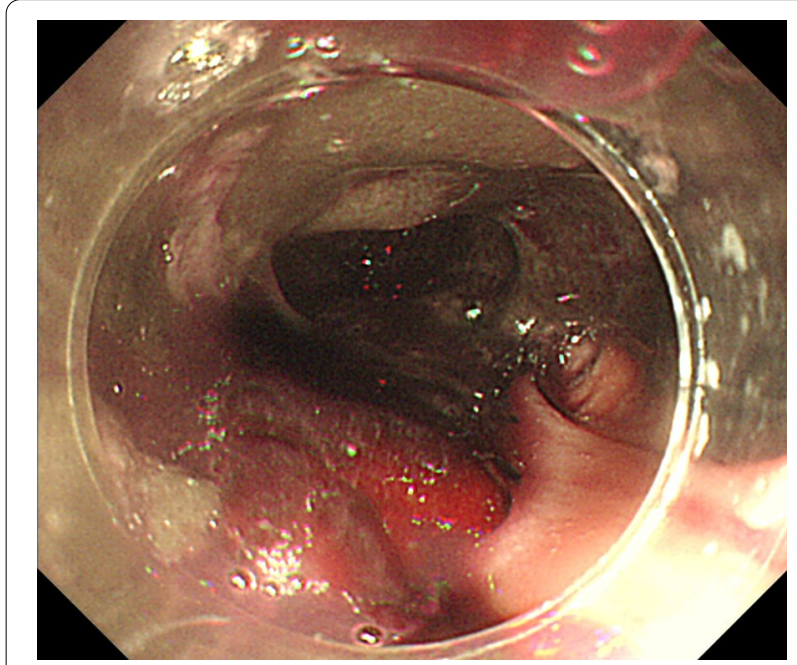

Fig. 7 Gastroscopy showing cytomegalovirus esophagitis

cancer and one case of triple cancer were noted. The types of cancer included lung $(n=7)$, breast $(n=5)$, gastric $(n=5)$, colon $(n=1)$, esophageal $(n=1)$, urothelial $(n=1)$, pancreatic $(n=1)$, thyroid $(n=1)$, thymic $(n=1)$, ovarian ( $n=1$ case), extragonadal germ cell tumor $(n=1)$, and myelodysplastic syndrome $(n=1)$. Creatine kinase values differed widely. Our case reported the maximum value of creatine kinase $(15,667 \mathrm{U} / \mathrm{L})$ among all cases in the literature. The option for treatment showed surgical treatment to internal treatment in five cases, whereas the opposite was true in seven cases. Internal treatment only was performed in nine cases, and surgical treatment only was performed in one case. Regarding response to internal treatment, "partial response" was defined as temporary remission of the symptoms and creatine kinase level only to worsen later. "No response" was defined as progressive symptoms over time. Remission was noted in five cases and partial response was observed in eight cases, while seven cases showed no response to the internal treatment.

As for treatment for DM, systemic steroid therapy is considered the gold standard. Oral prednisolone at an initial dose of $0.5-1 \mathrm{mg} / \mathrm{kg} /$ day followed by a slow progressive dose reduction is recommended. In patients with severe disease, steroid pulse of intravenous mPSL $1000 \mathrm{mg}$ for three consecutive days is also an option for treatment. In addition, the introduction of IVIG or immunosuppressive medications such as methotrexate, azathioprine, cyclophosphamide, or ciclosporin is another option if the patient does not respond to steroid therapy or suffers adverse side effects $[9,29]$. Although these internal treatments are essential for the control of DM, the risk of surgical treatment will increase due to the immunocompromised effect. Since tapering of the prednisolone dose takes a relatively long time, the timing of surgery before or after internal treatment is important. In our case, surgical treatment was delayed due to uncontrollable dysphagia and longterm use of steroid therapy. The treatment for cancer in the case reports included surgical resection and chemoradiotherapy. Immune checkpoint inhibitors such as nivolumab and ipilimumab were reported in three cases $[22,23,26]$. One report noted that only surgical removal of the tumor resulted in the disappearance of the skin rash of DM [15]. 


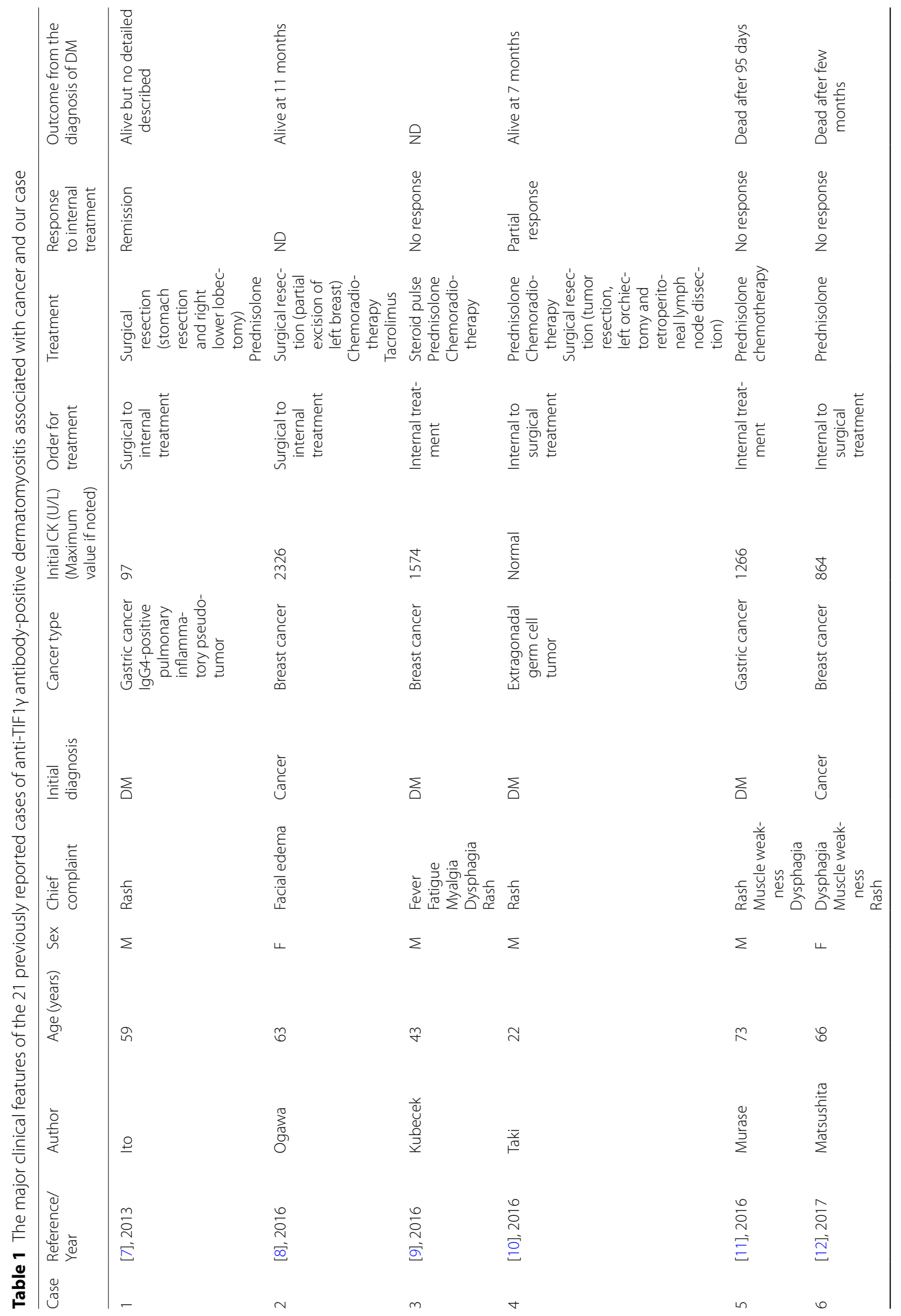




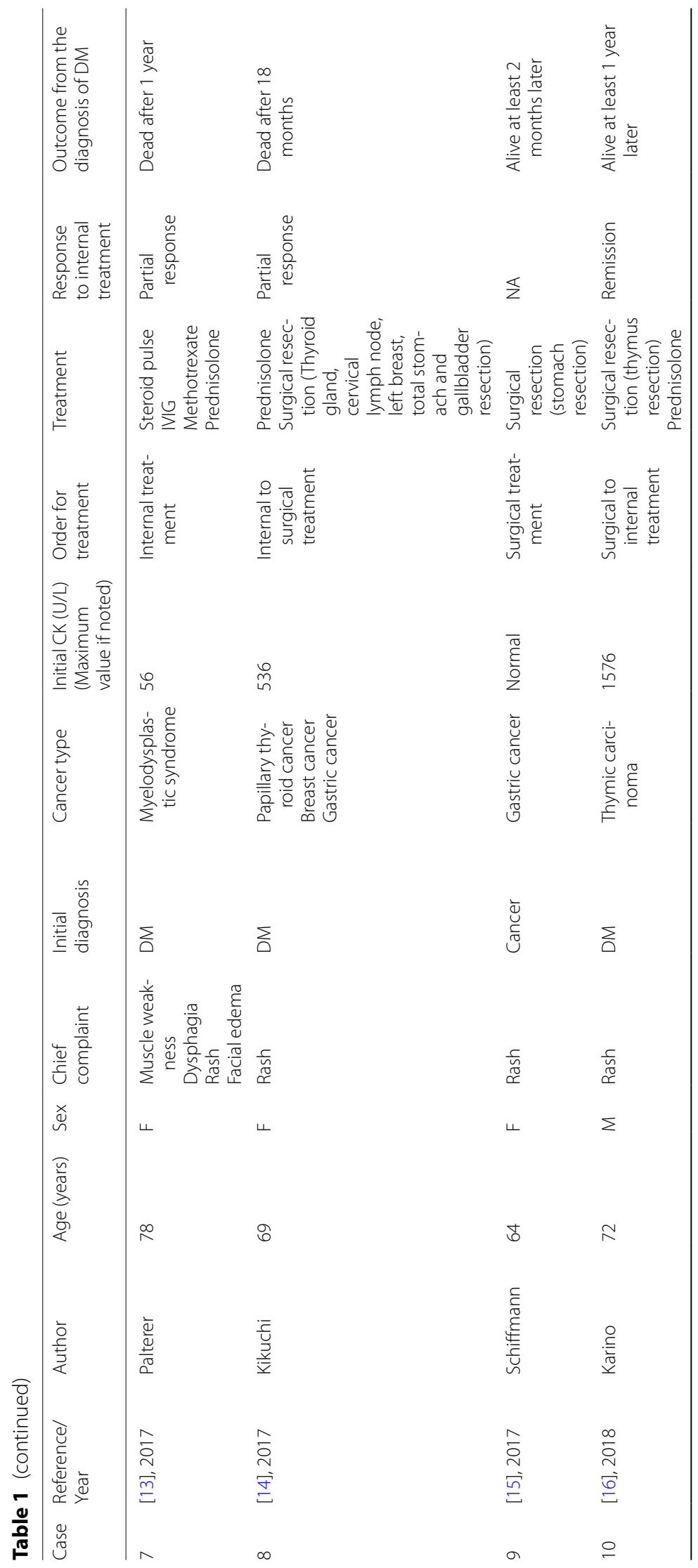




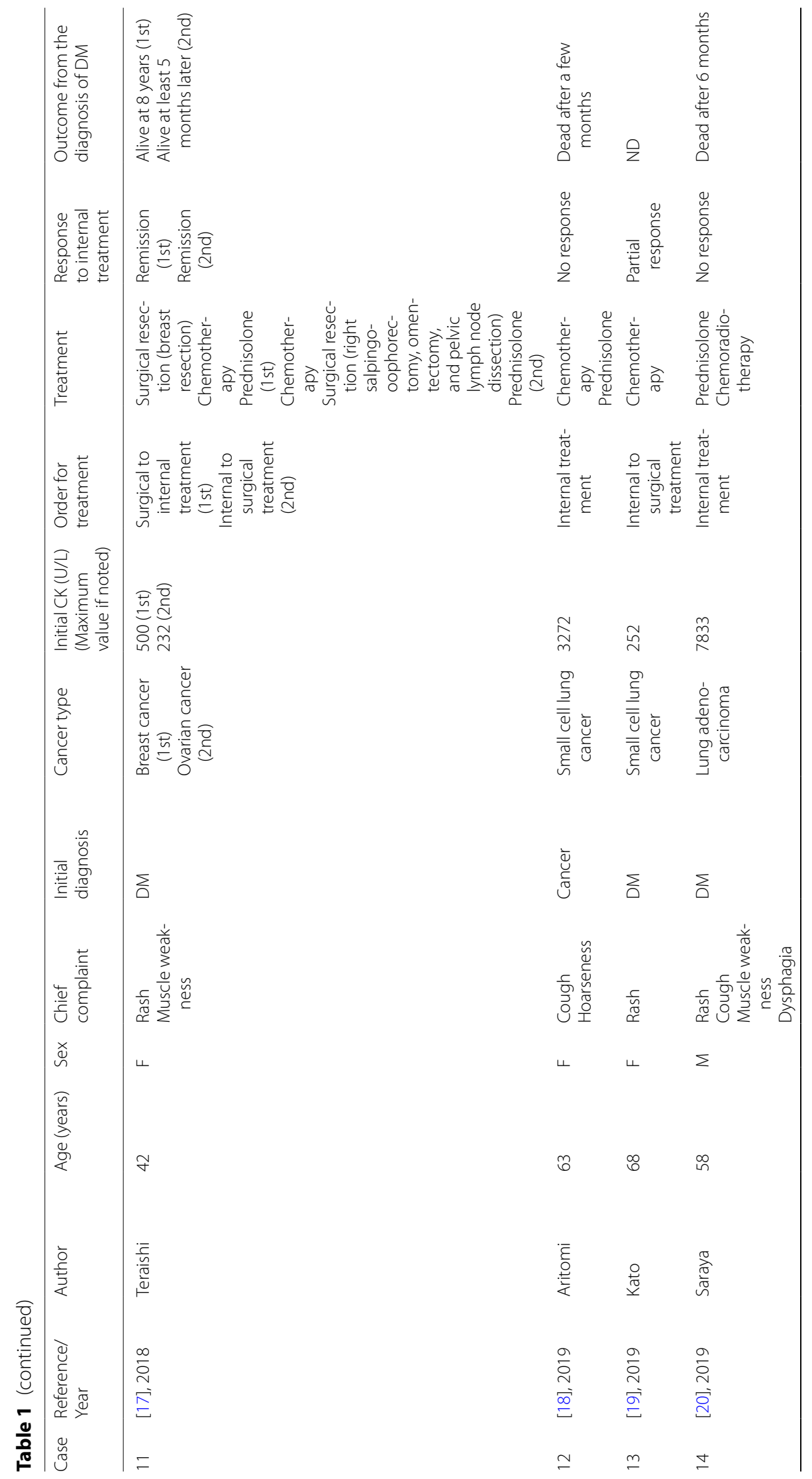




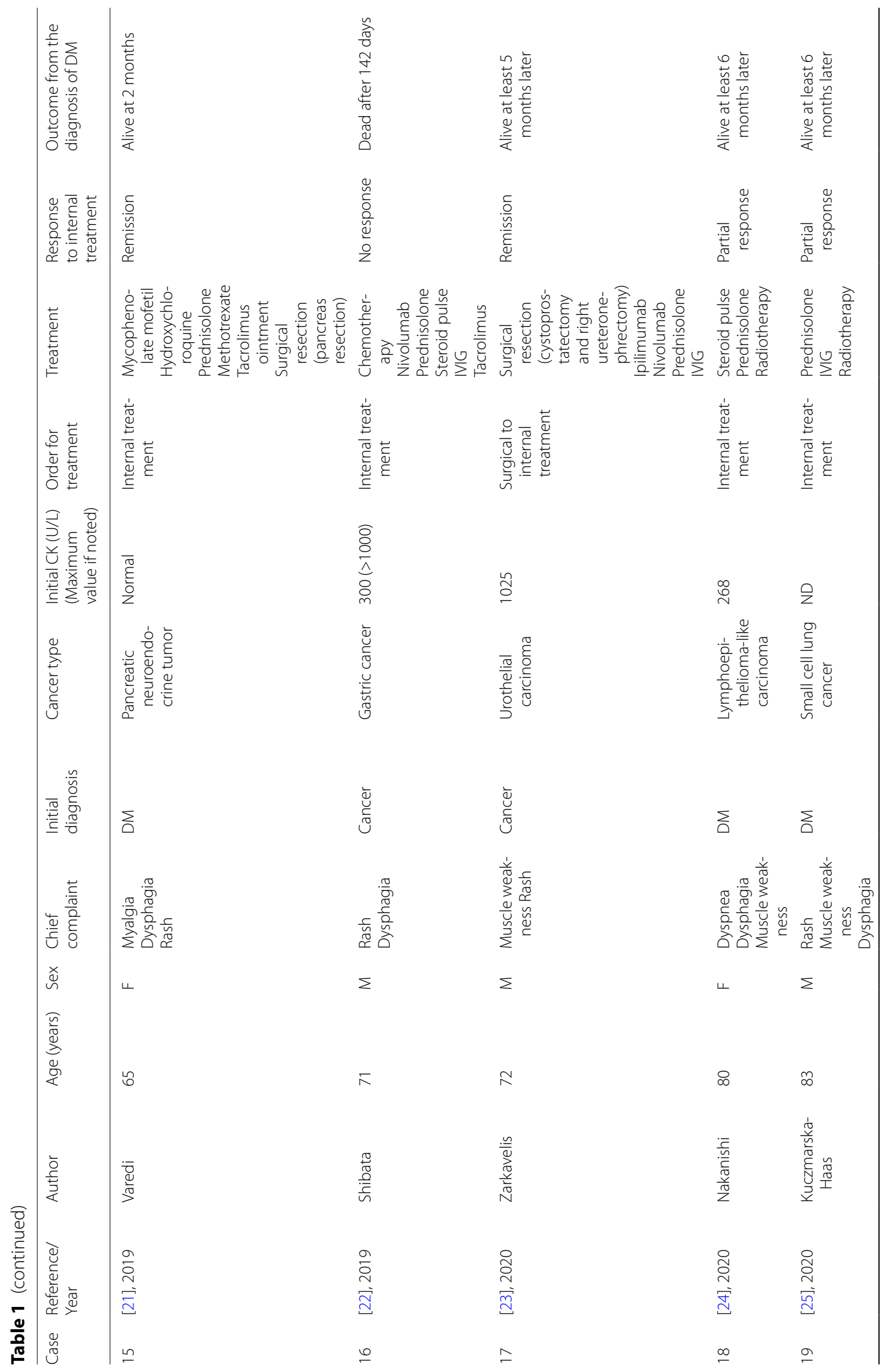




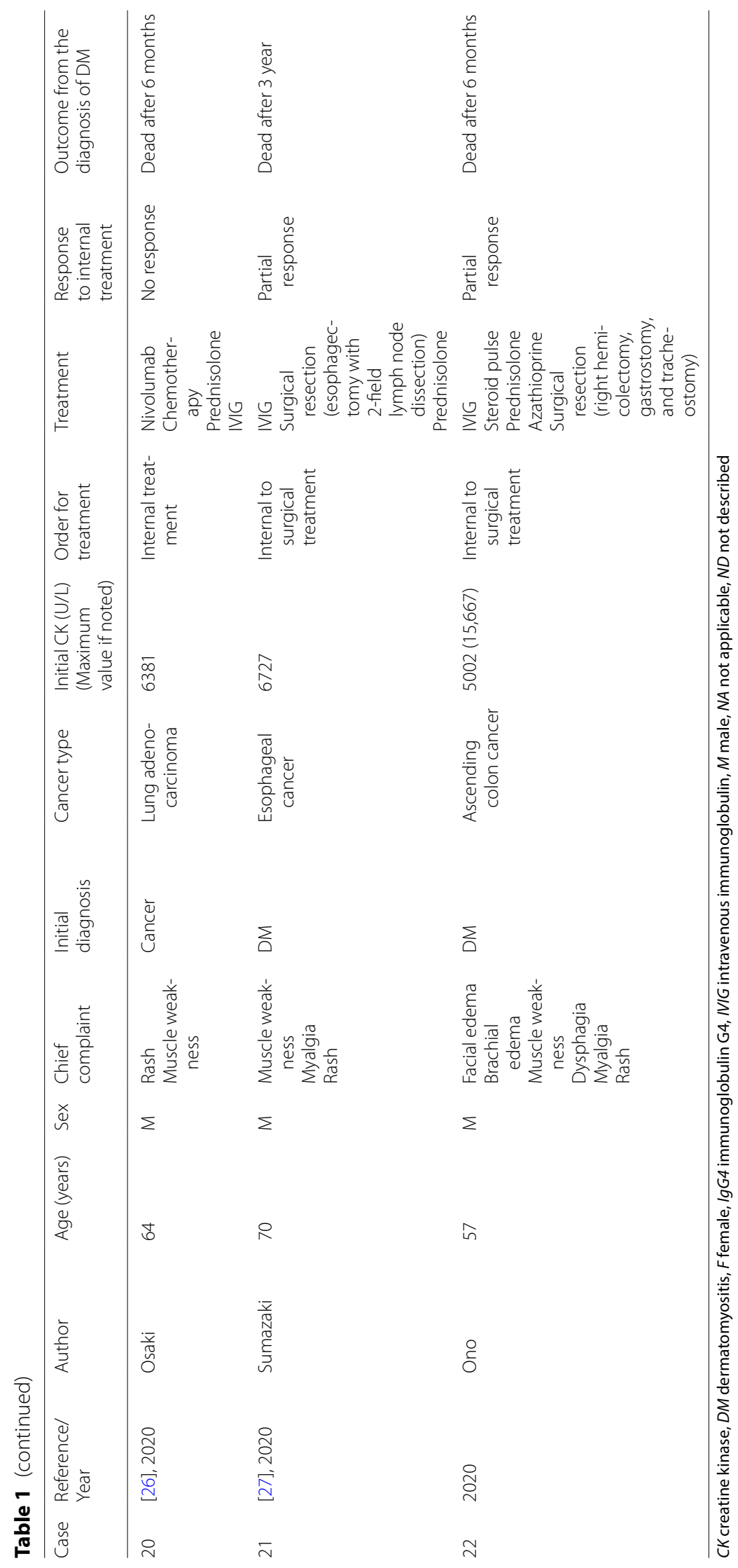


The outcomes revealed that half of the patients were alive while the others had died. We further investigated the relationship between outcome and dysphagia, initial treatment, and maximum creatine kinase values. Table 2 demonstrates this relationship. First, dysphagia is a major complication of DM because it leads to oral feeding difficulties and malnutrition [22]. Table 2 (a) shows the relationship between dysphagia and outcome; no significant difference $(P=0.12$; Pearson's chi-square test) was noted between them, although the presence of dysphagia tended to be associated with a worse outcome. Table 2 (b) shows the relationship between initial treatment and outcome. Of note, initial surgical treatment led to better outcomes $(P=0.0007$; Pearson's chi-square test $)$. However, interpretation must consider potential bias, as patients who are able to undergo surgery may have better general condition. In fact, patients with early-stage disease could firstly undergo surgery according to the cancer stage in Table 1. Conversely, the patients with initial internal treatment include unresectable cancers, in which surgical resection itself is not applicable at the point of diagnosis $[9,13,20,22,24,26]$. Table 2 (c) shows the relationship between the maximum creatine kinase level and outcome. In all four cases where the level was greater than $5000 \mathrm{U} / \mathrm{L}$, the patients died $(P=0.033$; Pearson's chisquare test). Lastly, the relationship between response to internal treatment and outcome is shown in Table 2 (d). In five of the cases with remission, the patients were

Table 2 The relationship between outcomes and the presence of dysphagia (a), the type of initial treatment (b), maximum creatine kinase level (c), and response to internal treatment (d)

\begin{tabular}{|c|c|c|c|}
\hline & \multicolumn{3}{|l|}{ Dysphagia } \\
\hline & $(+)$ & \multicolumn{2}{|l|}{$(-)$} \\
\hline Alive & 3 & \multicolumn{2}{|l|}{7} \\
\hline Dead & 7 & \multicolumn{2}{|l|}{4} \\
\hline \multicolumn{4}{|l|}{ (b) } \\
\hline & \multicolumn{3}{|c|}{ Initial treatment } \\
\hline & Surgical & \multicolumn{2}{|l|}{ Internal } \\
\hline Alive & 6 & \multicolumn{2}{|l|}{4} \\
\hline Dead & 0 & \multicolumn{2}{|l|}{10} \\
\hline \multicolumn{4}{|l|}{ (c) } \\
\hline & \multicolumn{3}{|c|}{ Max creatine kinase (U/L) } \\
\hline & $<5000$ & \multicolumn{2}{|l|}{$>5000$} \\
\hline Alive & 9 & \multicolumn{2}{|l|}{0} \\
\hline Dead & 6 & \multicolumn{2}{|l|}{4} \\
\hline & \multicolumn{3}{|c|}{ Response to internal treatment } \\
\hline & Remission & Partial & No response \\
\hline Alive & 5 & 3 & 0 \\
\hline Dead & 0 & 4 & 6 \\
\hline
\end{tabular}

alive, and in the group with no response, six patients had died. Among those with partial response, three patients were alive and four had died. These results suggest that a response to internal treatment is needed for lifesaving results.

\section{Conclusions}

In conclusion, we reviewed and summarized previously reported cases of anti-TIF1 $\gamma$ antibody-positive DM with malignancy. Cancer screening is essential in patients with anti-TIF1 $\gamma$ antibody-positive dermatomyositis because it is associated with a high prevalence of malignancies. Our review revealed that initial surgical treatment should be recommended for better prognosis if the general condition allows.

\section{Abbreviations}

DM: Dermatomyositis; IVIG: Intravenous immunoglobulin; TIF1Y: Transcriptional intermediary factor 1 gamma; mPSL: Methylprednisolone; MRI: Magnetic resonance imaging.

\section{Acknowledgements}

We would like to thank Editage [http://www.editage.com] for editing and reviewing this manuscript for English language.

\section{Authors' contributions}

$\mathrm{RO}$ was a major contributor to the writing of the manuscript and patient management. TK, MI and MY contributed to patient management. TM and KI critically revised the report and approved the final report. All authors read and approved the final manuscript.

\section{Funding}

None.

Availability of data and materials

Not applicable.

Ethics approval and consent to participate

Not applicable.

\section{Consent for publication}

Written informed consent was obtained from the patient's next of kin for publication of this case report and any accompanying images. A copy of the written consent is available for review by the Editor-in-Chief of this journal.

\section{Competing interests}

The authors state that they have no conflicts of interest.

\section{Author details}

1 Department of General Internal Medicine, Shonan Kamakura General Hospital, 1370-1 Okamoto, Kamakura, Kanagawa 247-8533, Japan. ${ }^{2}$ Department of Surgery, Shonan Kamakura General Hospital, 1370-1 Okamoto, Kamakura, Kanagawa 247-8533, Japan. ${ }^{3}$ Department of Rheumatology, Shonan Kamakura General Hospital, 1370-1 Okamoto, Kamakura, Kanagawa 247-8533, Japan.

Received: 18 October 2020 Accepted: 6 January 2021

Published online: 22 March 2021

References

1. Dalakas MC, Hohlfeld R. Polymyositis and dermatomyositis. Lancet. 2003:362(9388):971-82 
2. Kamiyama H, Niwa K, Ishiyama S, et al. Ascending colon cancer associated with dermatomyositis which was cured after colon resection. Case Rep Gastroenterol. 2016;10(2):338-43.

3. Yanagi T, Kato N, Yamane N, Osawa R. Bullous pemphigoid associated with dermatomyositis and colon carcinoma. Clin Exp Dermatol. 2007;32(3):291-4.

4. Andras C, Ponyi A, Constantin T, et al. Dermatomyositis and polymyositis associated with malignancy: a 21 -year retrospective study. J Rheumatol. 2008;35(3):438-44.

5. Hida A, Yamashita T, Hosono Y, et al. Anti-TIF1-gamma antibody and cancer-associated myositis: a clinicohistopathologic study. Neurology. 2016;87(3):299-308

6. Trallero-Araguas E, Rodrigo-Pendas JA, Selva-O'Callaghan A, et al. Usefulness of anti-p155 autoantibody for diagnosing cancer-associated dermatomyositis: a systematic review and meta-analysis. Arthritis Rheum. 2012;64(2):523-32.

7. Ito K, Imafuku S, Hamaguchi Y, Fujimoto M, Nakayama J. Case report of anti-transcription intermediary factor-1-gamma/alpha antibody-positive dermatomyositis associated with gastric cancer and immunoglobulin G4-positive pulmonary inflammatory pseudotumor. J Dermatol. 2013;40(7):567-9.

8. Ogawa M, Sugiura K, Yokota K, Muro Y, Akiyama M. Anti-transcription intermediary factor 1-gamma antibody-positive clinically amyopathic dermatomyositis complicated by interstitial lung disease and breast cancer. J Eur Acad Dermatol Venereol. 2016;30(2):373-5.

9. Kubecek O, Soukup T, Paulik A, Kopecky J. Dermatomyositis with anti-TIF1 gamma antibodies as a presenting symptom of underlying triple-negative breast cancer: a case report. BMC Cancer. 2016;16(1):684.

10. Taki E, Shimizu M, Soeda Y, Shirai M, Muro Y. Anti-TIF1-gamma-positive young adult dermatomyositis with germ cell tumour. Eur J Dermatol. 2016:26(6):623-4.

11. Murase C, Muro Y, Nakanishi H, Katsuno M, Akiyama M. A patient with Parkinson's disease and dermatomyositis with serum anti-transcriptional intermediary factor 1-gamma antibody. Eur J Dermatol. 2016;26(6):617-8.

12. Matsushita T, Takehara K, Oishi K, et al. Case of anti-transcriptional intermediary factor-1-positive dermatomyositis associated with breast cancer developing over 10 years. J Dermatol. 2017;44(8):972-3.

13. Palterer B, Vitiello G, Cammelli D. First report of anti-TIF1gamma dermatomyositis in a patient with myelodysplastic syndrome. Reumatismo. 2017:69(2):75-7.

14. Kikuchi N, Ohashi T, Miura T, Nishibu A, Yamamoto T. Triple cancers concurrently detected in a case of antitranscriptional intermediary factor-1gamma antibody-positive dermatomyositis. Int J Dermatol. 2017;56(12):1516-7.

15. Schiffmann ML, Warneke VS, Ehrchen J. Amyopathic dermatomyositis with anti-TIF1 gamma antibodies. J Dtsch Dermatol Ges. 2018:16(1):76-8.

16. Karino K, Fujieda Y, Kawamura T, et al. Anti-TIF1 gamma antibody predicted malignancy of thymic tumor with dermatomyositis as an "autoimmune tumor marker": A case report. Medicine (Baltimore). 2018;97(49):e13563.

17. Teraishi M, Nakajima K, Ishimoto T, et al. Anti-transcription intermediary factor 1 gamma antibody titer correlates with clinical symptoms in a patient with recurrent dermatomyositis associated with ovarian cancer. Int J Rheum Dis. 2018:21(4):900-2.
18. Aritomi T, Kido T, Nakano K, et al. Small cell lung cancer patient with antitranscriptional intermediary factor 1 gamma antibody who developed dermatomyositis after successful chemoradiotherapy. Intern Med. 2019;58(3):427-31.

19. Kato T, Ito S, Tsuzuki T, Watanabe D, Kubo A, Yamaguchi E. Small cell lung cancer and interstitial pneumonia associated with anti-transcriptional intermediary factor-1 gamma-positive dermatomyositis. Respirol Case Rep. 2019;7(4):e00412.

20. Saraya T, Tamura M, Kasuga K, Fujiwara M, Takizawa H. Lung adenocarcinoma and anti-transcriptional intermediary factor 1-gamma positive dermatomyositis complicated with spontaneous oesophageal rupture. Respirol Case Rep. 2019;7(3):e00403.

21. Varedi D, Frigerio A, Scaife C, Hull C. A novel case of TIF1 gamma autoantibody positive dermatomyositis associated with a non-functional pancreatic neuroendocrine tumor. Dermatol Online J. 2019;25(3):13030.

22. Shibata C, Kato J, Toda N, et al. Paraneoplastic dermatomyositis appearing after nivolumab therapy for gastric cancer: a case report. J Med Case Rep. 2019;13(1):168

23. Zarkavelis G, Mauri D, Karassa F, et al. The cancer immunotherapy environment may confound the utility of anti-TIF-1gamma in differentiating between paraneoplastic and treatment-related dermatomyositis. Report of a case and review of the literature. ContempOncol (Pozn). 2020;24(1):75-8.

24. Nakanishi Y, Yamaguchi K, Yoshida Y, et al. Coexisting TIF1 gamma-positive primary pulmonary lymphoepithelioma-like carcinoma and anti-TIF1 gamma antibody-positive dermatomyositis: a case report. Intern Med. 2020. https://doi.org/10.2169/internalmedicine.4702-20.

25. Kuczmarska-Haas A, Burr AR, Witt JS, et al. Dramatic improvement of anti-transcription intermediary factor-1-gamma/alpha antibody-positive dermatomyositis after stereotactic body radiotherapy to presumed lung primary-a case report. PractRadiatOncol. 2020. https://doi.org/10.1016/j. prro.2020.06.008

26. Osaki M, Tachikawa R, Ohira J, Hara S, Tomii K. Anti-transcriptional intermediary factor 1-gamma antibody-positive dermatomyositis induced by nivolumab for lung adenocarcinoma: a case report. Invest New Drugs. 2020. https://doi.org/10.1007/s10637-020-00974-7.

27. Sumazaki M, Kaneko K, Ito M, et al. A case of dermatomyositis along with esophageal cancer and screening of serum transcriptional intermediary factor 1 gamma antibodies in various cancer patients. Am J Case Rep. 2020;21:e922004.

28. Aussy A, Boyer O, Cordel N. Dermatomyositis and immune-mediated necrotizing myopathies: a window on autoimmunity and cancer. Front Immunol. 2017;8:992

29. Marie I, Mouthon L. Therapy of polymyositis and dermatomyositis. Autoimmun Rev. 2011;11(1):6-13.

\section{Publisher's Note}

Springer Nature remains neutral with regard to jurisdictional claims in published maps and institutional affiliations.

Ready to submit your research? Choose BMC and benefit from

- fast, convenient online submission

- thorough peer review by experienced researchers in your field

- rapid publication on acceptance

- support for research data, including large and complex data types

- gold Open Access which fosters wider collaboration and increased citations

- maximum visibility for your research: over $100 \mathrm{M}$ website views per year

At BMC, research is always in progress.

Learn more biomedcentral.com/submissions 